\title{
Examining Weight Bias among Practicing Canadian Family Physicians
}

\author{
Angela S. Alberga ${ }^{a} \quad$ Sarah Nutter $^{b} \quad$ Cara Maclnnis ${ }^{c} \quad$ John H. Ellard ${ }^{c}$ \\ Shelly Russell-Mayhew ${ }^{b}$ \\ aDepartment of Health, Kinesiology, and Applied Physiology, Concordia University, \\ Montreal, QC, Canada; b Werklund School of Education, University of Calgary, Calgary, AB, \\ Canada; ' Department of Psychology, University of Calgary, Calgary, AB, Canada
}

\section{Keywords}

Weight stigma $\cdot$ Healthcare $\cdot$ Family physicians · Obesity

\begin{abstract}
Objectives: The aim of this study was to examine the attitudes of practicing Canadian family physicians about individuals with obesity, their healthcare treatment, and perceptions of obesity treatment in the public healthcare system. Method: A national sample of Canadian practicing family physicians $(n=400)$ completed the survey. Participants completed measures of explicit weight bias, attitudes towards treating patients with obesity, and perceptions that people with obesity increase demand on the public healthcare system. Results: Responses consistent with weight bias were not observed overall but were demonstrated in a sizeable minority of respondents. Many physicians also reported feeling frustrated with patients with obesity and agreed that people with obesity increase demand on the public healthcare system. Male physicians had more negative attitudes than females. More negative attitudes towards treating patients with obesity were associated with greater perceptions of them as a public health demand. Conclusion: Results suggest that negative attitudes towards patients with obesity exist among some family physicians in Canada. It remains to be determined if physicians develop weight bias partly because they blame individuals for their obesity and its increased demand on the Canadian public healthcare system. More research is needed to better understand causes and consequences of weight bias among health professionals and make efforts towards its reduction in healthcare.

(c) 2019 The Author(s)

Published by S. Karger AG, Basel
\end{abstract}


Alberga et al.: Examining Weight Bias among Practicing Canadian Family Physicians

\section{Introduction}

Weight bias represents negative attitudes and beliefs about individuals because of their weight. Negative attitudes and beliefs about individuals who are classified as overweight and obese are pervasive in the general public [1] and in healthcare professionals. Many health professionals, including physicians [2], nurses [3], dietitians [4], and mental health professionals [5], have been shown to hold weight bias, which can result in individuals with obesity avoiding routine or preventative healthcare [2]. It has been shown that weight bias is present even in healthcare providers specializing in care for people living with overweight and obesity $[5,6]$.

Much of the information we have about weight bias in healthcare comes from studies of students in postsecondary training to become future health professionals [7, 8]. Medical students have high levels of both implicit (unconscious) and explicit (conscious) weight bias at the start of medical school [8], and many are unaware of their bias related to weight [7]. Studies of US physicians in practice have found that $1 / 3$ characterized patients with obesity as sloppy and lazy [9], 45\% had a negative attitude toward individuals with obesity [10], and only $56 \%$ perceived themselves as competent to treat obesity in their medical practice [10]. Moreover, implicit and explicit weight bias were positively related in a study of physicians, indicating that relative to other types of prejudice (e.g., race, sexuality, disability) weight bias is more socially acceptable [2]. This research is especially troubling as weight bias may impact a patient's quality of healthcare $[11,12]$.

The present investigation adds to this body of scholarship by examining weight bias in a national sample of family physicians in Canada. To our knowledge, this is the first study to examine weight bias in a large sample of practicing family physicians across Canada. Family physicians were chosen because they are most often the first point of contact in healthcare [13]. In addition to assessing weight bias, the study examined the relationships between weight bias, attitudes about treating patients with obesity, and how people with obesity are perceived as a burden to the public healthcare system. This is of particular interest given the public healthcare system in Canada. As a potential source of concern for Canadian physicians, we sought to examine if perceiving obesity as a threat to the public healthcare system would be related to weight bias.

Our study also examined other potentially influential variables: gender and respondent body mass index (BMI). Existing research has found that women (vs. men) report less weight bias, but still within the range of higher weight bias [14]. Doctors who were classified as having obesity according to their BMI demonstrated moderate implicit weight bias, but doctors with underweight, normal, or overweight BMIs had high explicit and implicit bias [2].

\section{Materials and Methods}

\section{Participants}

A probability sample of 400 currently practicing family physicians completed the survey by phone or online. Participants were recruited from a contact list of 22,615 Canadian physicians of all types purchased by the survey research firm that collected the data. Provinces were sampled with a goal of achieving proportionate provincial representation. Most provinces were proportionately represented according to recent data for number of physicians by province [15] with 2 exceptions: participants from Alberta were overrepresented $(11.68 \%$ of population; $17.8 \%$ of participants) and participants from Quebec were underrepresented (22.95\% of population; $6 \%$ of participants). Participant gender, age, ethnicity, and province 
Alberga et al.: Examining Weight Bias among Practicing Canadian Family Physicians

Table 1. Participant demographics

\begin{tabular}{|c|c|c|}
\hline Variable & $n$ & $\%$ \\
\hline \multicolumn{3}{|l|}{ Gender } \\
\hline Male & 245 & 61.3 \\
\hline Female & 151 & 37.8 \\
\hline Preferred not to disclose & 4 & 1.0 \\
\hline \multicolumn{3}{|l|}{ Ethnicity } \\
\hline Asian & 25 & 6.3 \\
\hline Black & 32 & 8.0 \\
\hline White & 253 & 63.3 \\
\hline First Nations & 10 & 2.5 \\
\hline Hispanic & 6 & 1.5 \\
\hline Middle Eastern & 30 & 7.5 \\
\hline Pacific Islander & 2 & 0.5 \\
\hline Southeast Asian & 11 & 2.8 \\
\hline South Asian & 18 & 4.5 \\
\hline Biracial & 4 & 1.0 \\
\hline Other & 9 & 2.3 \\
\hline \multicolumn{3}{|l|}{ Province } \\
\hline Alberta & 71 & 17.8 \\
\hline British Columbia & 53 & 13.3 \\
\hline Manitoba & 11 & 2.8 \\
\hline New Brunswick & 10 & 2.5 \\
\hline Newfoundland and Labrador & 6 & 1.5 \\
\hline Nova Scotia & 10 & 2.5 \\
\hline Ontario & 146 & 36.5 \\
\hline Prince Edward Island & 2 & 0.5 \\
\hline Quebec & 24 & 6.0 \\
\hline Saskatchewan & 9 & 2.3 \\
\hline Yukon & 2 & 0.5 \\
\hline Declined to answer & 56 & 14.0 \\
\hline
\end{tabular}

Percentages were rounded to the nearest tenth.

of practice are reported in Table 1. Self-reported height and weight were reported by 208 participants $\left(\mathrm{M}_{\mathrm{BMI}}=25.77, \mathrm{SD}=4.76\right)$. This study was part of a larger study aimed to examine the association between considering obesity as a chronic disease and weight bias in a representative sample of Canadian family physicians [16] that was approved by the University of Calgary's research ethics board.

\section{Materials and Procedure}

After providing demographic information, participants completed the study measures. Explicit weight bias was measured using 9 items modified from the Universal Measure of Bias - Fat version [17] (UMB-Fat; $\alpha=0.73$; see Table 2 for all scale items). Seven items from the Attitudes about Treating Patients with Obesity scale [18] were used $(\alpha=0.74)$. These item responses were averaged with higher scores indicating greater weight bias or more negative attitudes about treating patients with obesity, respectively. Three items created by the researchers measured the extent to which participants believed that obesity is a burden to the public healthcare system. These items were examined separately given lower internal consistency $(\alpha=0.57)$. All items were assessed on 1 (strongly disagree) to 7 (strongly agree) scales. 
Table 2. Frequency of low, moderate, and high scores on attitude measures

\begin{tabular}{|c|c|c|c|c|c|}
\hline & $\begin{array}{l}\text { Disagree } \\
\text { (responses of } \\
1-3), n(\%)\end{array}$ & $\begin{array}{l}\text { Scale midpoint } \\
\text { (responses of } \\
4), n(\%)\end{array}$ & $\begin{array}{l}\text { Agree } \\
\text { (responses of } \\
5-7), n(\%)\end{array}$ & $\begin{array}{l}\text { Missing, } \\
n(\%)\end{array}$ & Means (SD) \\
\hline \multicolumn{5}{|l|}{ Universal Measure of Bias - Fat } & 2.95 (1.17) \\
\hline People with obesity tend toward bad behavior & $234(58.5)$ & $86(21.5)$ & $66(16.5)$ & $14(3.5)$ & $2.81(1.62)$ \\
\hline People with obesity are sloppy & $231(57.8)$ & $89(22.3)$ & $71(17.8)$ & $9(2.3)$ & $2.96(1.54)$ \\
\hline Sometimes I think that people with obesity are dishonest & $256(64.0)$ & $64(16.0)$ & $67(16.8)$ & $13(3.3)$ & $2.63(1.58)$ \\
\hline People with obesity have bad hygiene & $237(59.3)$ & $90(22.5)$ & $60(15.0)$ & $13(3.3)$ & $2.91(1.50)$ \\
\hline \multicolumn{6}{|l|}{$\begin{array}{l}\text { In general, people with obesity don't think about the needs of other } \\
\text { people }\end{array}$} \\
\hline I would not want to have a person with obesity as a roommate & $213(53.3)$ & $115(28.8)$ & $58(14.5)$ & $14(3.5)$ & $3.02(1.53)$ \\
\hline I like people with obesity ${ }^{a}$ & $90(22.5)$ & $142(35.5)$ & $144(36.0)$ & $24(6)$ & $4.39(1.40)$ \\
\hline I don't enjoy having a conversation with a person with obesity & $251(62.8)$ & $86(21.5)$ & $51(12.8)$ & $12(3)$ & $2.76(1.60)$ \\
\hline \multicolumn{5}{|l|}{$\begin{array}{l}\text { I would be comfortable having a person with obesity in my group of } \\
\text { friends }^{\mathrm{a}}\end{array}$} & $4.90(1.70)$ \\
\hline $\begin{array}{l}\text { I would like having a person with obesity at my place of worship or } \\
\text { community center }^{\mathrm{a}}\end{array}$ & $80(20.0)$ & $119(29.8)$ & $174(43.5)$ & $27(6.8)$ & $4.65(1.56)$ \\
\hline \multicolumn{6}{|l|}{ Attitudes about Obesity and Public Healthcare System } \\
\hline $\begin{array}{l}\text { People with obesity increase demand on the public healthcare system } \\
\text { Any extra demand on the public healthcare system from people with }\end{array}$ & $106(26.5)$ & $91(22.8)$ & $198(49.5)$ & $5(1.3)$ & $4.50(1.55)$ \\
\hline obesity is unfair to people who are not obese & $225(56.3)$ & $98(24.5)$ & $71(17.8)$ & $6(1.5)$ & $3.02(1.58)$ \\
\hline $\begin{array}{l}\text { People with obesity should pay extra for access to the public health- } \\
\text { care system. }\end{array}$ & $233(58.3)$ & $106(26.5)$ & $53(13.3)$ & $8(2)$ & $2.84(1.58)$ \\
\hline \multicolumn{5}{|l|}{ Attitudes about Treating Patients with Obesity } & $3.29(1.05)$ \\
\hline I often feel frustrated with patients who have obesity & $150(37.5)$ & $114(28.7)$ & $133(33.3)$ & $3(0.8)$ & $3.76(1.59)$ \\
\hline I dislike treating patients with obesity & $242(60.5)$ & $84(21.0)$ & $71(17.8)$ & $3(0.8)$ & $2.86(1.59)$ \\
\hline \multicolumn{6}{|l|}{$\begin{array}{l}\text { I feel that patients with obesity are often noncompliant with } \\
\text { treatment recommendations }\end{array}$} \\
\hline I feel disgust when treating a patient with obesity & $245(61.3)$ & $76(19.0)$ & 74 (18.5) & $5(1.3)$ & $2.78(1.61)$ \\
\hline Treating patients with obesity is professionally rewarding ${ }^{\mathrm{a}}$ & $95(23.8)$ & $108(27.0)$ & $184(46.0)$ & $13(3.3)$ & $4.41(1.55)$ \\
\hline It is difficult to feel empathy for a patient with obesity & $227(56.8)$ & $102(25.5)$ & $64(16.0)$ & $7(1.8)$ & $2.92(1.59)$ \\
\hline $\begin{array}{l}\text { Treating a patient with obesity is more stressful than treating a } \\
\text { nonobese patient }\end{array}$ & $179(44.8)$ & $97(24.3)$ & $116(29.0)$ & $8(2.0)$ & $3.58(1.61)$ \\
\hline
\end{tabular}

\section{Results}

Most respondents were white (63.3\%) men (61.3\%) aged 45 years or older (Table 1). The average total score of explicit weight bias was 2.95 (1.17) evaluated on a 7-point Likert scale (Table 2). Whereas the majority of physicians surveyed did not indicate responses consistent with a higher weight bias (defined for our purposes as agree responses [strongly, moderately, or somewhat] on typical items and disagree responses [strongly, moderately, or somewhat] on reverse coded items), a nontrivial minority did (a consistent 10-24\%; Table 2). For example, approximately $24 \%$ of physicians sampled indicated disliking of or discomfort with friendship/acquaintanceship with people with obesity. About $50 \%$ of the sample agreed that people with obesity increase demand on the public healthcare system, 33.3\% indicated feeling frustrated with patients who have obesity, $27.8 \%$ believed that patients with obesity are often noncompliant with treatment recommendations, and $18.5 \%$ indicated feeling disgusted when treating a patient with obesity.

Women (vs. men) reported less weight bias on UMB $(t(386)=3.95, p<0.001)$ and attitudes about treating patients $(t(392)=2.21, p=0.027)$. These relationships held when statistically controlling for ethnicity (White vs. non-White), region of residence, and age range using a multiple regression approach $(\beta=-0.23, p<0.001$ and $\beta=-0.16, p=0.005$, respectively) but became nonsignificant when also controlling for BMI ( $\beta=-0.08, p=0.320$ and $\beta=$ $0.09, p=0.242$, respectively). 
Men (vs. women, $t(387)=2.12, p=0.034$ ) as well as those with low BMIs (at/under $1 \mathrm{SD}$, $t(43)=2.88, p=0.006$ ) or medium BMIs (within $+/-1 \mathrm{SD}, t(182)=3.40, p=0.001$ ) versus high BMIs (at/above $1 \mathrm{SD}$ ) agreed more that those with obesity should pay extra for healthcare. When examining these associations using a regression approach, the relationship between gender and perceiving that people with obesity should pay more for healthcare remained when statistically controlling for ethnicity and region $(\beta=-0.15, p=0.009)$ but became nonsignificant when also controlling for BMI ( $\beta=0.01, p=0.927)$. The relationship between BMI and perceiving that people with obesity should pay more for healthcare was no longer significant when statistically controlling for gender, region, age range, and ethnicity $(\beta=-0.11, p=0.164)$.

Those with medium versus high BMIs also scored higher on the item assessing whether people with obesity increase demand on the public healthcare system $(t(184)=2.28, p=$ $0.001)$ and weight bias $(t(183)=2.33, p=0.021)$. These latter (but not former) differences were also present when using Center for Disease Control and Prevention (CDC) cutoffs for defining underweight, normal weight, overweight, and obesity [19] $(t(124)=1.95, p=0.053$ and $t(123)=2.00, p=0.048$, respectively).

Higher weight bias (UMB) was associated with more negative attitudes toward treating patients with obesity $(r=0.66)$, greater perceptions that extra demand on the public healthcare system from people with obesity is unfair $(r=0.61)$, and greater agreement that people with obesity should pay extra for public healthcare $(r=0.68)$. More negative attitudes toward treating patients with obesity were associated with greater perceptions that people with obesity increase demand on public healthcare $(r=0.13)$, greater perceptions that extra demand on the public healthcare system from people with obesity is unfair $(r=0.60)$, and greater agreement that people with obesity should pay extra for public healthcare $(r=0.64$, $p s<0.009$ ). All of these associations remained when statistically controlling for participant gender, ethnicity, age range, and region ( $p s<0.031$ ).

\section{Discussion/Conclusion}

This is the first study to report on weight bias in a large sample of currently practicing family physicians in Canada. Although weight bias was not present in the majority of the sample, it was present among some physicians, consistent with previous evidence from the US $[2,8]$. It is promising that the majority of physicians did not indicate higher scores of weight bias. However, approximately 6.3 million adult Canadians are living with obesity [20]. Our results suggest then that, even though only a minority of physicians are weight biased, many patients with obesity may see doctors who consider them disgusting, sloppy, dishonest, or unlikeable. Given the potential negative implications for care of patients living with obesity, it is critical to reduce weight bias among physicians. Our study also showed that weight bias was higher among men and those with lower BMIs, consistent with previous studies [14].

Unique to the Canadian context is the publicly funded healthcare system. Interestingly, half of practicing physicians agreed that people with obesity increased the demand on the public healthcare system, which was related to more weight bias and worse attitudes about treating patients with obesity. Public health demand items were associated with feeling frustrated with patients with obesity $(r s=0.32-0.33$, $p s<0.001)$. The causal model for these relationships is unclear and it remains to be determined if some physicians working in a publicly funded healthcare system may develop weight bias in part because they blame individuals with obesity for the increased demand that obesity and its associated comorbidities place on the Canadian public healthcare system.

This study emphasizes the need to reduce weight bias among practicing physicians in addition to making efforts to sensitize students entering the medical profession. Without such 
initiatives, patients with obesity may continue to experience harmful effects of weight bias, including less respect, less time spent with patients, and less patient-centered communication, thus contributing to negative effects on their overall health $[11,12]$.

\section{Conclusion}

This study contributes to our emerging understanding of weight bias among Canadian family physicians but is limited by the underrepresentation of family physicians in Quebec. Future work is needed to investigate weight bias reduction techniques targeted at physicians. Longitudinal studies may be informative to track and understand how weight bias manifests during formative medical training and how this may translate into practice over time. Future research investigating weight bias among Canadian samples of physicians within other medical specialties (e.g., bariatric surgery, obstetrics, gynecology) and other healthcare providers (e.g., exercise specialists, dietitians) would contribute valuable information about the environment of Canadian healthcare for people with obesity.

\section{Acknowledgement}

The authors gratefully acknowledge the Canadian family physicians who participated in this study.

\section{Statement of Ethics}

Participants have given their written informed consent. The study protocol has been approved by the University of Calgary's research ethics board.

\section{Disclosure Statement}

The authors have no conflicts of interest to declare.

\section{Funding Sources}

This research was funded by a University of Calgary Research Grants Council Grant. Dr. Alberga is currently supported by a Research Scholar Junior 1 Award from les Fonds de Recherche du Québec-Santé at Concordia University, Montreal, and was previously funded by a Banting Postdoctoral Fellowship Award from the Canadian Institutes of Health Research at the University of Calgary. Ms. Nutter is currently funded by a Doctoral Fellowship from the Social Sciences and Humanities Research Council of Canada.

\section{Author Contributions}

All authors conceived the study idea and designed the study. C.M. conducted the statistical analyses. All authors interpreted the findings. A.S.A. drafted the first version of the manuscript. All authors contributed writing, editing, and approval of the final draft submitted. 


\section{References}

1 Hansson LM, Rasmussen F. Attitudes towards obesity in the Swedish general population: the role of one's own body size, weight satisfaction, and controllability beliefs about obesity. Body Image. 2014 Jan;11(1):43-50.

2 Sabin JA, Marini M, Nosek BA. Implicit and explicit anti-fat bias among a large sample of medical doctors by BMI, race/ethnicity and gender. PLoS One. 2012;7(11):e48448.

3 Brown I. Nurses' attitudes towards adult patients who are obese: literature review. J Adv Nurs. 2006 Jan; 53(2):221-32.

4 Stone 0, Werner P. Israeli dietitians' professional stigma attached to obese patients. Qual Health Res. 2012 Jun;22(6):768-76.

5 Puhl RM, Latner JD, King KM, Luedicke J. Weight bias among professionals treating eating disorders: attitudes about treatment and perceived patient outcomes. Int J Eat Disord. 2014 Jan;47(1):65-75.

6 Michael Vallis T, Currie B, Lawlor D, Ransom T. Healthcare professional bias against the obese: how do we know if we have a problem? Can J Diabetes. 2007;31(4):365-70.

7 Miller DP Jr, Spangler JG, Vitolins MZ, Davis SW, Ip EH, Marion GS, et al. Are medical students aware of their anti-obesity bias? Acad Med. 2013 Jul;88(7):978-82.

8 Phelan SM, Burgess DJ, Burke SE, Przedworski JM, Dovidio JF, Hardeman R, et al. Beliefs about the causes of obesity in a national sample of 4th year medical students. Patient Educ Couns. 2015 Nov;98(11):1446-9.

9 Foster GD, Wadden TA, Makris AP, Davidson D, Sanderson RS, Allison DB, et al. Primary care physicians' attitudes about obesity and its treatment. Obes Res. 2003 Oct;11(10):1168-77.

10 Jay M, Kalet A, Ark T, McMacken M, Messito MJ, Richter R, et al. Physicians' attitudes about obesity and their associations with competency and specialty: a cross-sectional study. BMC Health Serv Res. 2009 Jun; 9(1):106.

11 Kirk SF, Price SL, Penney TL, Rehman L, Lyons RF, Piccinini-Vallis H, et al. Blame, Shame, and Lack of Support: A Multilevel Study on Obesity Management. Qual Health Res. 2014 Jun;24(6):790-800.

12 Phelan SM, Burgess DJ, Yeazel MW, Hellerstedt WL, Griffin JM, van Ryn M. Impact of weight bias and stigma on quality of care and outcomes for patients with obesity. Obes Rev. 2015 Apr;16(4):319-26.

13 Canadian Institutes of Health Information [Internet]. Primary health care data and information. 2018 [cited 2018 Mar 30]. Available from: https://www.cihi.ca/en/info_phc_data_en.pdf

14 Puhl RM, Latner JD, O’Brien K, Luedicke J, Danielsdottir S, Forhan M. A multinational examination of weight bias: predictors of anti-fat attitudes across four countries. Int J Obes. 2015 Jul;39(7):1166-73.

15 Canadian Medical Association [Internet]. Number of physicians by province/territory and specialty. 2017 [cited 2019 Oct 31]. Available from: https://www.cma.ca/sites/default/files/2019-03/2017-01-spec-prov. pdf

16 MacInnis CC, Alberga AS, Nutter S, Ellard JH, Russell-Mayhew S. Regarding obesity as a disease is associated with lower weight bias among physicians: A cross-sectional survey study. Stigma Health. 2019, https://doi. org/10.1037/sah0000180.

17 Latner JD, O’Brien KS, Durso LE, Brinkman LA, MacDonald T. Weighing obesity stigma: the relative strength of different forms of bias. Int J Obes. 2008 Jul;32(7):1145-52.

18 Puhl RM, Luedicke J, Grilo CM. Obesity bias in training: attitudes, beliefs, and observations among advanced trainees in professional health disciplines. Obesity (Silver Spring). 2014 Apr;22(4):1008-15.

19 Centers for Disease Control and Prevention [Internet]. Defining Adult Overweight and Obesity. 2019 [cited 2019 June 14]. Available from: https://www.cdc.gov/obesity/adult/defining.html

20 Statistics Canada [Internet]. Adjusting the scales: Obesity in the Canadian population after correction for respondent bias. [cited 2018 July 4]. Available from: https://www150.statcan.gc.ca/n1/pub/82624-x/2014001/article/11922-eng.htm 